\title{
Antimicrobial Effect
}

National Cancer Institute

\section{Source}

National Cancer Institute. Antimicrobial Effect. NCI Thesaurus. Code C40858.

An Antimicrobial Effect involves interference with, or restraint of, the activities of biologic molecules and complexes, or cellular components involved in the life cycle of a microorganism, resulting in suppression of microbial growth. Mechanisms to suppress growth include inhibition of cell wall synthesis; increased cell wall permeability; protein synthesis inhibition; and inhibition of reverse transcriptase and protease. 